\title{
Diez años del caracol gigante africano en Colombia: Revisión de la investigación y divulgación desarrollada entre 2008-2017
}

\author{
Angie Patiño Montoya ${ }^{12,}$ \& $\&$ Alan Giraldo ${ }^{1}$ \\ 1 Grupo de Investigación en Ecología Animal, Departamento de Biología, Facultad de Ciencias Naturales y Exactas, \\ Universidad del Valle, Cali, Colombia. ${ }^{2}$ Doutorado em Ecología, Programa de Pós-graduação em Ecología, Instituto de \\ Ciencias Biológicas, Universidade de Brasilia, Brasilia, Brasil.
}

\begin{abstract}
Resumen. La presencia del caracol gigante africano (Achatina fulica) como especie exótica en Colombia desde 2008 ha impulsado alertas sociales, acciones de control y esfuerzos de investigación sobre diferentes aspectos de la historia natural bajo las condiciones ambientales del territorio nacional. Con el propósito de consolidar el conocimiento generado y divulgado acerca del caracol gigante africano en Colombia durante los 10 años de presencia, se buscó información en las principales bases de datos científicas y públicas a nivel nacional e internacional. Esta información se filtró según la fuente, el año, la zona y el tema, y se analizó la variación temporal de los reportes de presencia y control. La mayor parte de la información disponible proviene de los medios de comunicación. Se establecieron los años 2014 y 2016 como los de mayor divulgación, predominando temas de manejo y control, seguidos por los registros de presencia. De acuerdo con la cronología de la información consolidada se soporta la hipótesis de la entrada del molusco al país. Se encontró una relación entre la frecuencia de los registros de presencia con procesos climáticos de escala interanual (e.g., ENOS) e intra-anual (e.g., variación estacional asociado a la dinámica temporal de la zona de convergencia intertropical). Además, se detectó que aunque se le dio una prioridad mayor a las estrategias de control mediado por la comunidad, no se generó aún suficiente conocimiento científico sobre las características biológicas y ecológicas de la especie, en relación con las condiciones ambientales del país.
\end{abstract}

[Palabras clave: Achatina fulica, estrategias de control, invasión biológica]

\begin{abstract}
Aвstract. Ten years of the African giant snail in Colombia: Review of the research and dissemination carried out between 2008-2017. The presence of the Giant African Snail (Achatina fulica) as an exotic species in Colombia since 2008 has promoted social alerts, control actions and research efforts on different aspects of natural history under the environmental conditions of the national territory. With the purpose of consolidating the knowledge that has been generated and disseminated on the Giant African Snail in Colombia during the 10 years of presence, information was sought in the main scientific and public databases at national and international level. This information was filtered according to the source, the year, the area and the subject, analyzing the temporal variation of the presence and control reports. This information comes from the media, establishing 2014 and 2016 as the years of greatest disclosure and predominant management and control issues, followed by presence records. According to the chronology of the consolidated information, the hypothesis of the entry of mollusk into the country is supported. A relationship was found between the frequency of presence records with interannual (e.g., ENSO) and intra-annual climatic processes (e.g., seasonal variation associated with the temporal dynamics of the intertropical convergence zone). In addition, it was found that although community-mediated control strategies have been given a higher priority, scientific knowledge on the adjustment of the biological and ecological characteristics of the species to the environmental conditions of the country has not been generated.
\end{abstract}

[Keywords: Achatina fulica, control strategies, biological invasion]

\section{INTRODUCCIÓN}

La eficacia de un plan de manejo y control para una especie exótica dependerá de la articulación de los diferentes actores en la sociedad afectada (Shackleton et al. 2019). A mayor escala espacial, más grande la dificultad en las interacciones academia-autoridad ambiental-comunidad para ejecutar acciones decontrol(Courchampetal.2016; Shackleton et al. 2019). En las escalas meso y macro espacial, la comunicación científica implica una serie de retos significativos, en especial cuando se trata de divulgar conocimiento para otros sectores de la sociedad con relación a especies exóticas

Editor asociado: Martín Nuñez 两 angie.patino@correounivalle.edu.co o introducidas (Geraldi et al. 2019; Leppanen et al. 2019). En este sentido, le corresponde a la comunidad científica permear la opinión pública sobre la problemática ambiental, lo que lleva a minimizar o maximizar los efectos ecológicos y sociales de una población exótica determinada (Leppanen et al. 2019).

El caracol gigante africano (Achatina fulica; Bowdich, 1822) es considerado una de las 100 especies invasoras más peligrosas a nivel mundial (Raut and Baker 2002). Es un animal atractivo y de fácil transporte que fue introducido en toda la zona tropical del

Recibido: 4 de Junio de 2019

Aceptado: 16 de Diciembre de 2019 
planeta (Raut and Baker 2002; Fontanilla 2010). Su tasa reproductiva elevada y su condición de generalista en cuanto a hábitos alimentarios provocaron pérdidas económicas considerables en cultivos agrícolas y ornamentales en las regiones que colonizó (Raut and Baker 2002). Además, implica un riesgo epidemiológico al ser un molusco hospedero de diversos patógenos, como los nematodos del genero Angiostrongylus, en especial Angiostrongylus cantonensis (Chen 1935), que puede causar meningitis eosinofílica en los seres humanos (Maldonado et al. 2012; Fontanilla et al. 2014; York et al. 2014; Giraldo et al. 2019). En Sur América, la presencia de este molusco se remonta a la década de 1980; fue introducido a la región a través de Brasil, desde donde colonizó el 70\% de los países de la región (Avendaño and Linares 2015; Miranda et al. 2015; Goldyn et al. 2016).

En Colombia, el caracol gigante africano fue clasificado como especie exótica invasora por el Ministerio de Ambiente y Desarrollo Sostenible mediante la resolución 0848 del 23 de mayo de 2008 (MAVDT 2008). Dos años después fue reportado por primera vez en el sur del país. Como medida de acción, en el 2011 se diseñó un plan de manejo basado en colecta manual, delegando a las autoridades ambientales regionales la responsabilidad no sólo de realizar las acciones de control, sino de promover el conocimiento necesario sobre la especie y realizar la promoción de la divulgación y apropiación social de este conocimiento (MAVDT 2011; De la OssaLacayo et al. 2012). Para 2012, el caracol gigante africano ya se encontraba registrado en la mitad del territorio nacional, alcanzando localidades de la costa caribe en el norte del país (De la Ossa-Lacayo et al. 2012).

La estrategia de control implementada, a partir de la colecta manual, depende en mayor medida de la detectabilidad de los individuos. Por lo tanto, resulta relevante conocer la relación de las poblaciones de este molusco terrestre con las condiciones ambientales, y en particular con la hidroclimatología de la región (Eduvirgemand Moreira 2018). En Colombia, el patrón nacional de precipitación es modulado principalmente por procesos de escala local asociados a la variabilidad estacional de la zona de convergencia intertropical (ZCTI), patrón que es afectado por procesos de mesoescala interanual como el ENOS (El Niño - Oscilación del Sur) (Poveda 2004). Junto con la geografía nacional, estos procesos producen variaciones intra-anuales en la precipitación en cada región geográfica, lo cual afectaría la abundancia del caracol, como se demostró para Paraná (Eduvirgem and Moreira 2019). Por otro lado, la generación de propuestas de control etnobotánico direccionadas hacia las acciones preventivas, que reducirían el problema de detectabilidad e identificación de los individuos, se encuentran en etapa de laboratorio. Por lo tanto, los experimentos realizados a la fecha con extractos de plantas sólo alcanzaron tasas de mortalidad del $20 \%$ y no se tiene certeza de su replicabilidad (Garcés-Restrepo et al. 2016; Patiño-Montoya and Giraldo 2018).

Al problema de la heterogeneidad ambiental colombiana se adiciona una sociedad que desconoce o niega el problema ambiental de la presencia del caracol gigante africano en los diferentes centros urbanos, lo que dificulta aun más el control basado en la colecta manual (Patiño-Montoya et al. 2019). Queda claro que las estrategias de control dirigidas por las autoridades ambientales en Colombia necesitan de un adecuado flujo de información entre los diferentes actores involucrados (academia, autoridad ambiental y comunidades) a través de los medios de comunicación (Courchamp et al. 2016). Por ello, en la presente investigación se revisa, por primera vez, el conocimiento generado y divulgado sobre el caracol gigante africano en Colombia durante su primera década en este territorio. Se destaca la importancia de estrechar las relaciones entre la generación del conocimiento científico y las capacidades de divulgación, socialización y apropiación social del mismo a través de los medios masivos de comunicación.

\section{Materiales y MÉTODOS}

\section{Búsqueda}

Se buscó información relacionada con el caracol gigante africano en las bases de datos de Google Scholar, EBSCO, Web of Science; también en Youtube, repositorios institucionales de las Universidades Colombianas, las Corporaciones Autónomas Regionales, archivos digitales de los periódicos de Colombia y fuentes radiales nacionales existente hasta junio de 2017. Para documentos en español se utilizaron las palabras clave caracol africano, caracol africano Colombia y caracol gigante africano. Las palabras Achatina fulica, Lissachatina fulica y Angiostrongylus se usaron para documentos en otros idiomas. 
El intervalo de tiempo usado dependió del alcance del motor de búsqueda.

\section{Clasificación}

Para facilitar el procesamiento, la interpretación y la independencia de la información se siguieron las recomendaciones de Colley y Fischer (2009), quienes hipotetizan que la baja accesibilidad de la información dificulta las estrategias de control y, por lo tanto, se recomienda, además de las publicaciones científicas, literatura gris en este tipo de revisiones. Los archivos conseguidos fueron clasificados de acuerdo con la fuente, por tema, tiempo y región administrativa (denominado como departamento). Se filtraron los documentos provenientes de los periódicos hasta fecha y localidad geográfica para evitar la replicación. Con base en la fuente de origen, se consideró un producto como medio de comunicación si su procedencia era periódicos, YouTube o fuente radial si correspondía a un archivo de audio. Se consideró como documento científico si su procedencia era una publicación en una revista o evento científico especializado en Colombia o sobre Colombia, como documento universitario si fueron trabajos de grado presentes en repositorios institucionales y finalmente documento gubernamental si fueron documentos derivados de actos legislativos del Ministerio de Ambiente, las Corporaciones Autónomas Regionales o gobernaciones.

La segunda clasificación consistió en el tema del documento. Para esto se establecieron cuatro categorías:a)controly manejo,b) reporte de presencia, c) historia natural y ecología, y d) genética y parasitología. Se consideró un producto como parte de la categoría control y manejo si el objetivo principal era dar pautas o formas de proceder en la manipulación o erradicación de la especie. Se consideró un producto como parte de la categoría reporte de presencia si el propósito del documento era indicar la aparición de individuos de la especie por primera vez o por reaparición en una localidad. Se consideró un producto como parte de la categoría historia natural y ecología si su propósito era divulgar aspectos sobre la biología de la especie o las interacciones con su entorno. Por último, se consideró un producto dentro de la categoría genética y parasitología si el documento contenía la descripción de aspectos moleculares de la especie o se encontraba relacionado con los parásitos de interés de salud pública asociados al caracol.

La tercera clasificación fue temporal. Para ello se agrupó toda la información teniendo en cuenta el año en que fue divulgada, considerando las regiones y todo el país. Finalmente, la información se clasificó por zona geográfica como región a) Caribe, b)

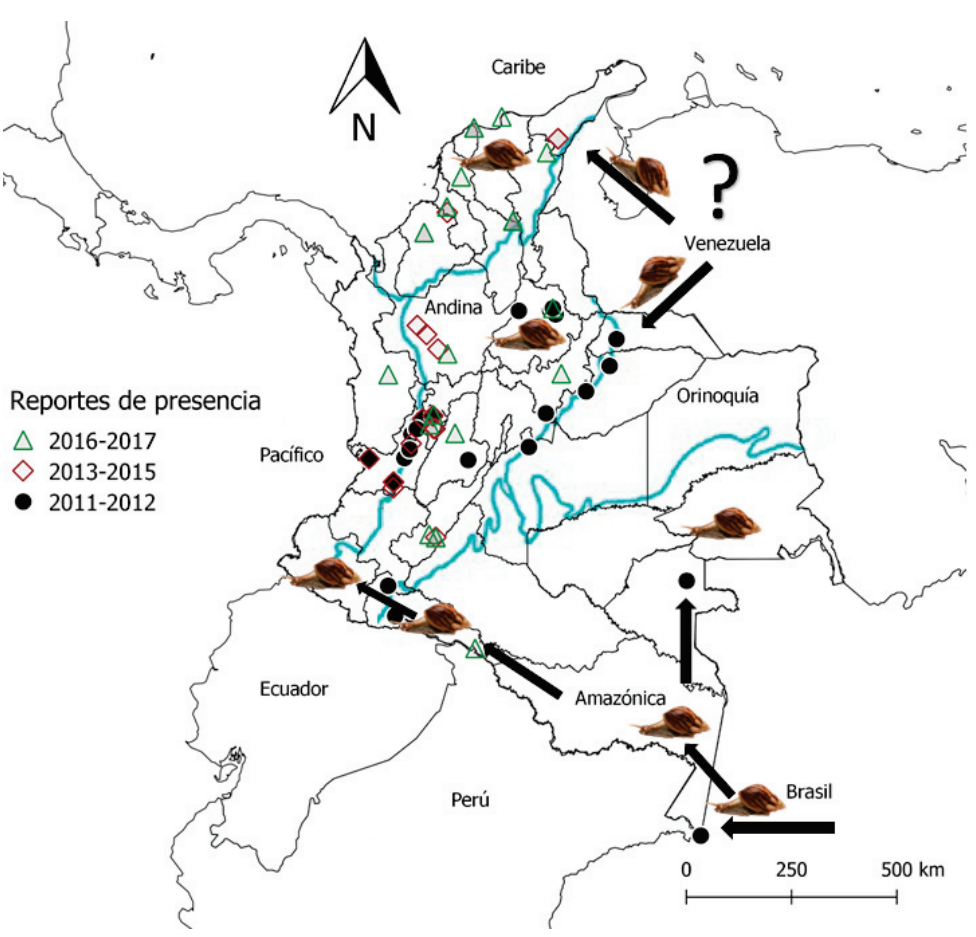

Figura 1. Reportes de presencia del caracol gigante africano (Achatina fulica) en cinco regiones naturales de Colombia: Caribe, Andina, Pacífico, Orinoquía y Amazónica entre el 2011 y 2017.

Figure 1. Reports of the presence of the African giant snail (Achatina fulica) in five natural regions of Colombia: Caribbean, Andean, Pacific, Orinoco and Amazonian between 2011 and 2017. 
Andina, c) Pacífica y d) Oriental, dependiendo del sitio de estudio o de reporte indicado en el documento. Los documentos de la Región Amazónica y Orinoquía fueron unificados como región Oriental (Figura 1). Esta clasificación fue realizada considerando los patrones espaciales y temporales de la temperatura del aire y precipitaciones reportados por Pabón-Caicedo et al. (2001), en los que cada región presenta distintos valores de temperatura media anual y patrones de precipitación cuasi monomodales, bimodales y monomodales, respectivamente, que pueden modular las poblaciones del caracol gigante africano. Además se consideró la mayor concentración de la población colombiana en los valles interandinos ubicados en la región Andina y en las ciudades de Cartagena, Santa Marta y Barranquilla en la región Caribe (Hernández 2010).

\section{Análisis estadísticos}

Con el propósito de realizar una descripción sinóptica de la información contenida, se tabularon las frecuencias y se construyeron gráficas por cada una de las clasificaciones. Además, sin incluir los documentos audiovisuales, se evaluó si la frecuencia de los reportes (presencia, manejo y control) por

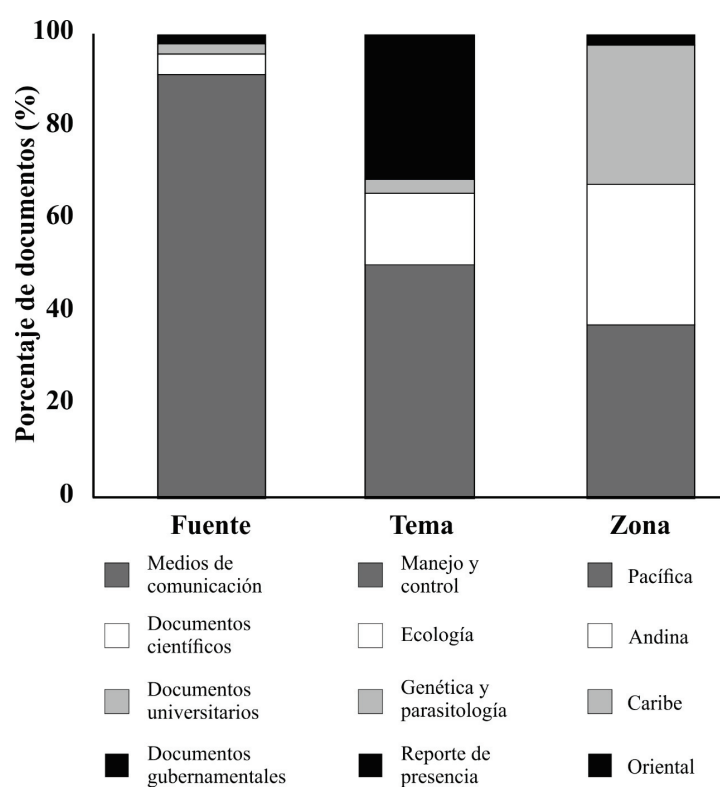

Figura 2. Porcentaje de documentos relacionados con el caracol gigante africano, según tipo de fuente. Documentos analizados: 261.

Figure 2. Percentage of documents related to the Giant African Snail, found by type of source. Documents analyzed: 261 región varían temporalmente por medio de un test exacto de Fisher en el programa R versión 3.4.2 (R Core Team 2018).

\section{Resultados}

Se recopilaron 261 archivos con información acerca del caracol gigante africano (Achatina fulica) en Colombia desde el 2008 hasta abril del 2017. La fuente que aportó el mayor cubrimiento de la información fueron los medios de comunicación escritos, radiales y visuales, con 239 documentos (Figura 2). El esfuerzo de investigación científica nacional dedicada a la generación de conocimiento sobre esta especie durante el período de estudio produjo 11 documentos, repartidos en 3 eventos científicos, un libro y 7 publicaciones en revistas especializadas (una en revista internacional, 5 en revistas nacionales y una publicación de impacto departamental). Por último, las organizaciones gubernamentales y las universidades presentaron 6 y 5 documentos, respectivamente.

Considerando el tipo de tema desarrollado por los documentos, el manejo y control obtuvo un mayor número de registros (100 documentos), seguido por los reportes de presencia (62 documentos en 45 localidades), mientras que la historia natural, ecología, genética y parasitología registraron en conjunto 37 documentos (Figura 2). Es importante mencionar que en 2010 se divulgaron dos reportajes en medios de comunicación, en los que se discute la relación del caracol gigante africano con la muerte de un bebé por meningitis eosinofílica como consecuencia de la exposición al parásito nemátodo Angiostrongylus cantonensis (Chen, 1935), que puede estar presente en esta especie exótica (Tabla 1).

Al analizar temporalmente la divulgación de la información generada sobre el caracol gigante africano en Colombia, se identificó una tendencia bimodal con puntos máximos (en 2014, con 41 documentos, y en 2016, con 56 documentos) y dos registros mínimos (en 2012, con 15 documentos, y en 2015, con 8 documentos). La información de manejo y control supera en todos los años la información biológica o ecológica, excepto en 2015. En promedio, la generación y divulgación de información ecológica entre 2011 y 2016 se mantuvo en cinco documentos por año, al contrario del control y manejo, que presentó un incremento en el número de documentos 
Tabla 1. Observaciones relevantes del comportamiento de la presencia de Achatina fulica proporcionadas por los medios masivos de comunicación.

Table 1. Relevant observations of the behavior of the Achatina fulica presence, provided by the mass media.

\begin{tabular}{|c|c|c|c|}
\hline Tipo & Año & Origen & \\
\hline \multirow[t]{2}{*}{ Ingreso } & Desde 1960 & Caribe & $\begin{array}{l}\text { https://www.elheraldo.co/tendencias/carrizal-pierde-uno-de- } \\
\text { sus-mas-queridos-congos-274985 }\end{array}$ \\
\hline & Desde 2000 & Caribe & $\begin{array}{l}\text { http://www.atlantico.gov.co/index.php/noticias-salud/3582- } \\
\text { autoridades-de-salud-alertan-sobre-presencia-del-caracol- } \\
\text { gigante-africano-en-el-atlantico }\end{array}$ \\
\hline Salud pública & $\begin{array}{l}\text { Víctimas fatales } \\
\text { meningitis } 2010\end{array}$ & Pacifico & $\begin{array}{l}\text { https://www.eltiempo.com/archivo/documento/MAM- } \\
5112779\end{array}$ \\
\hline \multirow[t]{7}{*}{$\begin{array}{l}\text { Primeros } \\
\text { reportes }\end{array}$} & En Buenaventura 2010 & Pacifico & $\begin{array}{l}\text { https://www.elpais.com.co/valle/alerta-por-peligro-de- } \\
\text { caracol-africano-en-el-puerto-de-buenaventura.html }\end{array}$ \\
\hline & En Meta 2010 & Oriental & $\underline{\text { http://www.eltiempo.com/archivo/documento/CMS- }}$ \\
\hline & En Bucaramanga 2011 & Andina & $\begin{array}{l}\text { https://www.eltiempo.com/archivo/documento/CMS- } \\
11538066\end{array}$ \\
\hline & En Barrancabermeja 2011 & Andina & $\begin{array}{l}\text { https://www.vanguardia.com/santander/barrancabermeja/ } \\
\text { un-segundo-presunto-caracol-africano-obligo-a-tomar- } \\
\text { medidas-en-barrancabermeja-LAVL129442 }\end{array}$ \\
\hline & Por Venezuela 2012 & Caribe & $\begin{array}{l}\text { https://laguajirahoy.com/2014/05/caracol-gigante-africano- } \\
\text { invade-la.html }\end{array}$ \\
\hline & En Antioquia 2012 & Andina & $\begin{array}{l}\text { http://historico.elmundo.com/portal/noticias/antioquia/un_ } \\
\text { africano_que_amenaza_a_antioquia.php\#.WJeOK1N96M8 }\end{array}$ \\
\hline & En Cartagena 2016 & Caribe & $\begin{array}{l}\text { https://www.eluniversal.com.co/cartagena/confirman- } \\
\text { presencia-del-caracol-gigante-africano-en-cartagena-237262- } \\
\text { FXEU345044 }\end{array}$ \\
\hline
\end{tabular}

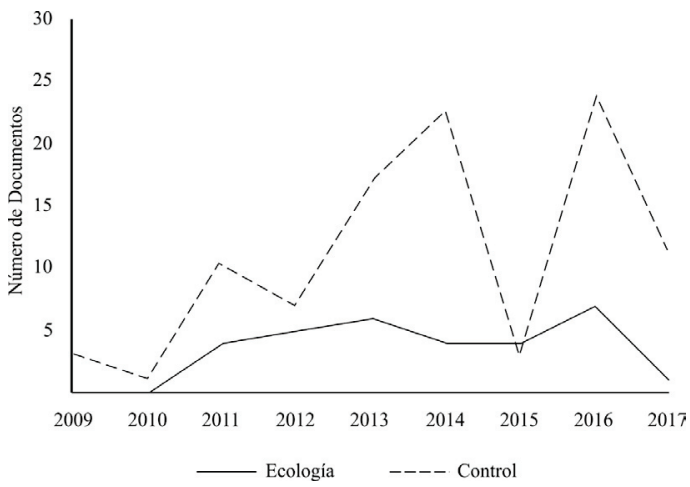

Figura 3. Número de documentos por año enfocados en los temas de 'ecología' y 'control del caracol gigante africano' (Achatina fulica) en Colombia.

Figure 3. Number of documents per year focused on the topics of 'ecology' and 'control of the Giant African Snail' (Achatina fulica) in Colombia.

generados entre 2012 y 2014, disminuyendo sustancialmente para el 2015. Sin embargo, para el 2016 la tendencia de número de documentos registrado alcanzó los niveles del 2014 (Figura 3).

No se encontraron registros documentales de caracol gigante africano en Colombia anteriores a 2010. Los primeros reportes de presencia se dieron entre el 2010 y el 2012 para todas las regiones por parte de los medios de comunicación, excepto la Región Oriental,
A 30

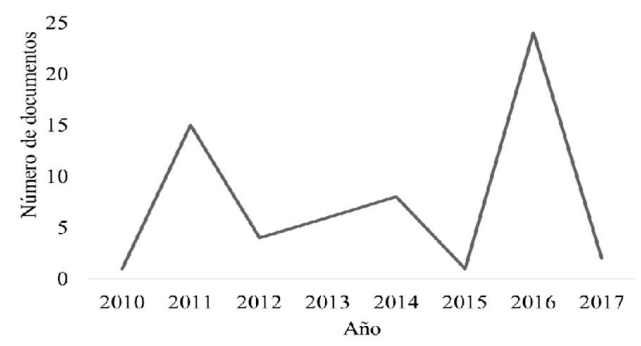

B

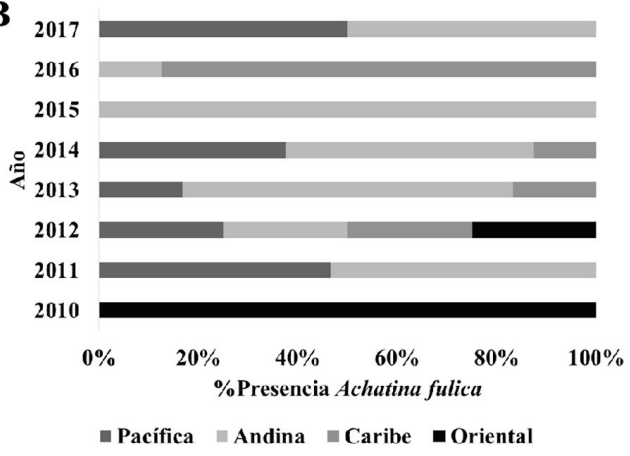

Figura 4. Cantidad de reportes de presencia de Achatina fulica por año, discriminando por región geográfica. A) Colombia, B) Regiones Pacífica, Andina, Caribe y Oriental.

Figure 4. Number of reports of the presence of Achatina fulica per year, discriminating by geographical region. A) Colombia, B) Pacific, Andean, Caribbean, Eastern regions. 
Tabla 2. Frecuencia de reportes de presencia, control y manejo de Achatina fulica en Colombia, en relación con la zona geográfica y temporalidad dada en años y cuatrimestres.

Table 2. Frequency of reports of presence, control and management of Achatina fulica in Colombia, in relation to the geographical area and temporality given in years and quarters.

\begin{tabular}{|c|c|c|c|c|c|}
\hline \multicolumn{6}{|c|}{ Reportes de presencia de Achatina fulica } \\
\hline Región & $2010-2013(\%)$ & $2014-2017(\%)$ & Ene-abr (\%) & May-ago (\%) & Sep-dic (\%) \\
\hline Pacífico & $12(75)$ & $4(25)$ & $12(75)$ & $2(12.5)$ & $2(12.5)$ \\
\hline Andina & $18(64.28)$ & $10(35.71)$ & 7 (29.16) & $6(25)$ & $11(45.83)$ \\
\hline Caribe & $4(17.39)$ & $19(82.60)$ & $3(15)$ & $7(35)$ & $10(50)$ \\
\hline Oriental & $3(100)$ & $0(0)$ & $1(50)$ & $0(0)$ & $1(50)$ \\
\hline \multicolumn{6}{|c|}{ Reportes de control y manejo Achatina fulica } \\
\hline Pacífico & 17 (55.55) & $32(65.30)$ & $25(55.55)$ & $12(26.66)$ & 8 (17.77) \\
\hline Andina & $13(38.46)$ & $19(59.37)$ & $10(38.46)$ & $10(38.46)$ & $6(23.07)$ \\
\hline Caribe & $5(23.80)$ & $20(80)$ & $5(23.80)$ & $1(4.76)$ & $15(71.42)$ \\
\hline Oriental & $4(62.5)$ & $3(42.85)$ & $5(62.5)$ & $1(12.5)$ & $2(25)$ \\
\hline$P$ valor & 0.21 & & & 0.0006 & \\
\hline
\end{tabular}

que fue por parte de la autoridad ambiental (Figura 1, Figura 4). Considerando la zona geográfica, la información disponible sobre la Región Oriental es escasa, con el registro de sólo 4 documentos, mientras que la región con mayor volumen de información fue la región Pacífica, particularmente el departamento del Valle del Cauca (con 65 documentos), seguida por la Región Andina (con 53) y la región Caribe (con 52). Por otro lado, la Región Oriental fue la primera en reportar el molusco, seguido por las regiones Pacífica y Andina, y la Región Caribe, que reportó al molusco en 2012 y para el 2016 obtuvo la mayor cantidad de reportes de presencias (Figura 4).

En cuanto al patrón temporal de la información, la zona geográfica y la temporalidad guardan una relación de dependencia, excepto en los reportes de control y manejo por año (Tabla 2). Para los reportes de presencia, se encontró que el mayor porcentaje de documentos para los departamentos hacia el sur fueron mayores entre 2010 y 2013 respecto a la región norte (Caribe), siendo el caso contrario para los años de 2014 y 2017 (Fisher, $P=0.00015)$. Por cuatrimestre, la zona Pacífica obtuvo un mayor porcentaje de documentos divulgados entre enero y abril, al contrario del resto del país (Fisher, $P=0.009$ ) (Figura 1, Tabla 2).

\section{DisCUSIÓN}

De acuerdo con Shackleton et al. (2019), el éxito de las estrategias de manejo y control de las especies exóticas involucra diferentes dimensiones, entre las que sobresalen la sociocultural y la gobernanza institucional. En este trabajo estarían permeando la percepción de la comunidad sobre la problemática, pues dependen de la disponibilidad de información científica adecuada. Aunque en Colombia, la información generada sobre el caracol gigante africano y su proceso de divulgación exhiben las mismas tendencias a lo establecido para Brasil (Colley and Fischer 2009; Fischer et al. 2015; Pavanelli et al. 2017), el volumen de la información científica disponible es escaso.

En términos generales, la poca información científica producida es el resultado de convenios interinstitucionales entre entidades universitarias y las autoridades ambientales regionales. Gracias a ello se generó algún conocimiento sobre la historia natural, genética y parasitología del caracol gigante africano en Colombia. Por otro lado, la alta presión política por controlar esta especie que ha tenido una rápida dispersión en el territorio nacional, direccionó a la comunidad científica nacional en reportes de presencia y control inmediato (De la Ossa-Lacayo et al. 2012; Giraldo et al. 2014). En consecuencia, la estrategia de control implementada por las autoridades ambientales regionales en Colombia estaría condenada al fracaso, ya que al parecer existe un déficit de información científica que requiere la comunidad y los tomadores de decisión.

A partir de la escasa investigación científica sobreel caracolgiganteafricano entre 2008-2017 en Colombia se pudo plantear una hipótesis sobre el ingreso de este molusco por el sur del país, dispersando hacia el norte. Además, se sugirió su relación directa con las cabeceras urbanas (De la Ossa-Lacayo et al. 2012; Giraldo et al. 2014), la variación de su abundancia con el régimen de precipitación en la región caribe (norte de Colombia) (De la Ossa et al. 2017), el registro del nematodo Angiostrongylus cantonensis en el país (Giraldo et al. 2019) 
e indicios de una respuesta poblacional de la morfología de la concha mediada por las acciones de control implementadas (Avendaño and Linares 2015; Patiño-Montoya et al. 2018). Aun así, las investigaciones con un enfoque social muestran una sociedad colombiana con negación ante el problema y, por ende, una baja receptividad ante las actividades de la autoridad ambiental (Patiño-Montoya et al. 2019).

Lamentablemente, el conocimiento generado por la ciencia colombiana no fue acogido por las autoridades ambientales o por los medios de comunicación, ya que la mayoría de los documentos son reportes de presencia. La baja asociación entre los medios de comunicación con la actividad y productos científicos es una constante para diferentes iniciativas de manejo, control o erradicación de especies exóticas, invasoras o introducidas a nivel mundial (Geraldi et al. 2019). Entonces, si las acciones de apropiación social del conocimiento a través de la divulgación han recaído casi que exclusivamente en los medios masivos de comunicación y la información que se divulga no necesariamente ha sido confirmada o no corresponde a las condiciones de desarrollo de las poblaciones en los ambientes colonizados, se estará reduciendo el alcance de los esfuerzos de apropiación social e identificación de la problemática ambiental por parte de las comunidades locales (Colley and Fisher 2009; Courchamp et al. 2016; Shackleton et al. 2019).

El análisis de la variación espacio-temporal de la información que fue recopilada para esta investigación apoya la hipótesis de dispersión sur-norte de Colombia por parte del caracol gigante africano, ya que la primera referencia bibliográfica sobre esta especie en el país, realizada por De la Ossa-Lacayo et al. (2012), indican que los primeros reportes de la especie ocurrieron durante 2010, mencionando entre los 15 departamentos con registros positivos de presencia los departamentos de la región sur del país: Amazonas, Putumayo y Nariño, hasta departamentos a $800 \mathrm{~km}$ al norte como Santander y Sucre. Esta información fue consistente con los reportes de presencia en cada zona por año, además de direccionarse a las zonas urbanas, situación similar a lo reportado en otros países de la región (Martínez-Escarbassiere et al. 2008; Correoso and Coello 2009; Vázquez et al. 2017).

Por otro lado, la variación temporal del número de reportes de presencia del caracol gigante africano por localidad parece estar relacionados con los regímenes de precipitación en el país. De acuerdo con el instituto de hidrología, meteorología y estudios ambientales de Colombia (IDEAM), 2011 y 2016 corresponden a años con registros de precipitación arriba del promedio histórico, mientras que 2015 fue un año seco (IDEAM 2017). En términos generales, bajo condiciones de alta humedad ambiental se reportó una tendencia al incremento en la abundancia y, por consiguiente, un aumento en el número de reportes de este molusco, mientras que, durante épocas secas, como en 2015, los reportes de avistamiento se hacen más esporádicos, tendencia que deben ser tenidas en cuenta cuando se están definiendo las acciones de control (Eduvirgem and Moreira 2018).

Aunque se identifican esfuerzos para generar un plan de manejo estructurado para el control del caracol gigante africano en Colombia, es preocupante la limitada producción científica sobre esta especie exótica, así como el limitado esfuerzo de sensibilización social sobre los potenciales impactos de la presencia de $A$. fulica en el país. Esto podría tener repercusiones en la eficiencia de las estrategias de control que se están implementando, toda vez que la participación de los diferentes actores sociales es fundamental para alcanzar las metas de control adecuadas. Por lo tanto, es urgente ampliar el horizonte de los esfuerzos de investigación realizados en el país sobre caracol gigante africano, para pasar de trabajos de presencia-ausencia a responder preguntas sobre la biología y ecología de la especie, que permitan incluso fortalecer las acciones de control. Además, se debe procurar incrementar las acciones de divulgación de la información que se genera de estos esfuerzos de investigación a través de estrategias de comunicación que permita estrechar los lazos entre los investigadores, tomadores de decisiones y el público general.

Agradecimientos. Al programa de Posgrado en Ciencias Biología de la Universidad del Valle por el estímulo académico de asistencia de docencia otorgado al primer autor. A Mario Fernando Garcés y el profesor Jaime Ricardo Cantera por las sugerencias realizadas durante la investigación, al comunicador social Jonathan Patiño por las sugerencias en la búsqueda dentro de los medios masivos de comunicación colombianos. A los diferentes revisores anónimos que con sus aportes contribuyeron a la mejora del manuscrito. 


\section{REFERENCIAS}

Avendaño, J. M., and E. Linares. 2015. Morfometría del caracol gigante africano Achatina fulica (Gastropoda: Achatinidae) en Colombia. Cuadernos de investigación 7(2):287-293. https:// doi.org/10.22458/urj.v7i2.1155.

Colley, E., and M.L. Fischer. 2009. Avaliação dos problemas enfrentados no manejo do caramujo gigante africano Achatina fulica (Gastropoda: Pulmonata) no Brasil. Zoologia 26(4):674-683. https://doi.org/10.1590/S198446702009000400012.

Correoso, M., and M. Coello. 2009. Modelación y distribución de Lissachatina fulica (Gastropoda: Achatinidae) en Ecuador. Potenciales impactos ambientales y sanitarios. Revista Geoespacial 6:79-90.

Courchamp, F., A. Fournier, C. Bellard, C. Bertelsmeier, E. Bonnaud, J. M. Jeschke, and J. C. Russell. 2016. Invasion biology: specific problems and possible solutions. Trends in Ecology and Evolution 32(1):13-22. https://doi.org/ 10.1016/j.tree.2016.11.001.

De la Ossa-Lacayo, A., J. De la Ossa, and C. Lasso. 2012. Registro del caracol africano gigante Achatina fulica (Bowdich 1822) (Mollusca: Gastropoda-Achatinidae) en Sincelejo, Costa Caribe de Colombia. Biota Colombiana 13(2):247252.

De La Ossa, J., A. De la Ossa-Lacayo, J. C. Carmona, M. M. Pineda, and J. F. Mario. 2017. Incremento poblacional de Achatina fulica Bowdich 1822 (mollusca: Gastropoda-Achatinidae) en una zona urbana de Sincelejo, Sucre, Colombia. ACCB 1(29):21-29. https:/ / doi.org/10.17485/ijst/2018/v11i21/123193.

Eduvirgem, R. V., and M. E. Moreira Costa Ferreira. 2018. Identificação do melhor período de controle de Achatina fulica em Maringá, Paraná, Brasil Vertices 21(1):109-124. https:/ / doi.org/10.19180/1809-2667.v21n12019p109-124.

Fischer, M. L., L. B. Caires, and E. Colley. 2015. Análise das informações veiculadas na internet sobre o Caramujo Gigante Africano Achatina fulica. Revista Brasileira de Pesquisa em Educação em Ciências 15(1):149-172.

Fontanilla, I. K. C. 2010. Achatina (Lissachatina) fulica BOWDICH: Its molecular phylogeny, genetic variation in global populations, and its possible role in the spread or the rat lungworm Angiostrongylus cantonensis (CHEN). Tesis doctoral. Universidad de Nottingham, Inglaterra. Pp. 800.

Fontanilla, I. K. C., I. M. P. S. María, J. R. M. García, H. Ghate, F. Naggs, and C. M Wade. 2014. Restricted genetic variation in populations of Achatina (Lissachatina) fulica outside of East Africa and the Indian Ocean Islands points to the Indian Ocean Islands as the earliest known common source. PloS ONE 9(9). https://doi.org/10.1371/ ournal.pone.0105151.

Garcés-Restrepo, M. F., A. Patiño-Montoya, M. Gómez-Díaz, A. Giraldo, and W. Bolívar-García. 2016. Sustancias alternativas para el control del caracol africano (Achatina fulica) en el Valle del Cauca, Colombia. Biota Colombiana 17(1):44-52. https://doi.org/10.21068/c2016v17r01a04.

Geraldi, N. R., A. Anton, C. E. Lovelock, and C. M. Duarte. 2019. Are the ecological effects of the "worst" marine invasive species linked with scientific and media attention? PLoS ONE 14(4):e0215691. https://doi.org/10.1371/ journal.pone.0215691.

Giraldo, A., W. Bolívar, and A. González (comp.). 2014. Caracol africano en el Valle del Cauca: línea base para el Valle del Cauca. Grupo de Investigación en Ecología Animal, Universidad del Valle. Cali. Pp. 19.

Giraldo, A., C. Garzón, A. Castillo, and D. F. Córdoba-Rojas. 2019. Confirmation of the presence of Angiostrongylus cantonensis in lung tissue of the African giant snail (Lissachatina fulica) in Colombia. Infectio 23(2):129-132. https: //doi.org/10.22354/in.v23i2.768.

Gołdyn, B., P. R. Guayasamín, K. A. Sánchez, and L. Hepting. 2016. Notes on the distribution and invasion potential of Achatina fulica Bowdich, 1822 (Gastropoda: Pulmonata: Achatinidae) in Ecuador. Folia Malacologica 24(2):85-90. https://doi.org/10.12657/folmal.024.014.

Ideam. 2017. Resumen mensual de la situación sinóptica. URL: https:/ / tinyurl.com/rpk4w4e.

Leppanen, C., D. M. Frank, J. J. Lockyer, C. J. Fellhoelter, A. K. Cameron, B. A. Hardy, and D. Simberloff. 2019. Media representation of hemlock woolly adelgid management risks: a case study of science communication and invasive species control. Biological Invasions 21(2):615-624. https:/ / doi.org/10.1007/s10530-018-1850-9.

Maldonado, A., R. Simões, and S. Thiengo. 2012. Angiostrongyliasis in the Americas. INTECH Open Access Publisher, Argentina. Pp. 303-320. https://doi.org/10.5772/38632.

Martínez-Escarbassiere, R., E. O. Martínez, O. Castillo, and L. Ruiz. 2008. Distribución geográfica de Achatina (Lissachatina) fulica (Bowdich, 1882) (Gastropoda-Stylommatophora-Achatinidae) en Venezuela. Memoria de la Fundación La Salle de Ciencias Naturales 169:93-106.

MAVDT. 2008. Resolución Número 0848 del 23 de mayo de 2008. Por el cual se declaran unas especies exóticas como invasoras y se señalan las especies introducidas irregularmente al país que pueden ser objeto de cría en ciclo cerrado y se adoptan otras determinaciones. Ministerio de Ambiente, Vivienda y Desarrollo Territorial. MAVDT, Colombia.

MAVDT. 2011. Resolución Número 654 de abril 7, 2011, por la cual se corrige la Resolución No. 0848 del 23 de mayo de 2008 y se adoptan las medidas que deben seguir las autoridades ambientales para la prevención, control y manejo de la especie Caracol Gigante Africano (Achatina fulica). MADTV, Colombia.

Miranda, M. S., J. H. Fontenelle, and I. L. Pecora. 2015. Population structure of a native and an alien species of snail in an urban area of the Atlantic Rainforest. Journal of natural history 49(1-2):19-35. https://doi.org/10.1080/ 00222933.2014.930756

Patiño-Montoya, A., and A. Giraldo, A. 2018. Valuation of alternative methodology for the control of the giant African snail (Achatina fulica). Boletín Científico Centro de Museos Museo de Historia Natural 22(2):183-192.

Patiño-Montoya, A., O. Murillo, and A. Giraldo. 2018. Variación morfológica poblacional de una especie invasora: el 
caracol gigante africano, Achatina fulica (Bowdich 1822) (Mollusca: Gastropoda-Achatinidae) en el departamento del Valle del Cauca, Colombia. Biota Colombiana 19(1):112-122. https:/ /doi.org/10.21068/2018.v19n01a07.

Patiño-Montoya, A., S. Giraldo-Ocampo, and A. Giraldo. 2019. Perception of giant African snail (Achatina fulica) in urban community from Colombia. Revista Facultad Nacional de Agronomía Medellín 72(1):8717-8727. https:// doi.org/10.15446/rfnam.v72n1.73085.

Pavanelli, G. C., M. U. Yamaguchi, E. A. Calaça, and F. H. Oda. 2017. Scientometrics of zoonoses transmitted by the giant African snail Achatina fulica Bowdich, 1822. Revista do Instituto de Medicina Tropical de São Paulo 59:1-7. https: //doi.org/10.1590/s1678-9946201759015.

Poveda, G. 2004. La hidroclimatología de Colombia: Una síntesis desde la escala interdecadal hasta la escala diurna. Revista de la Academia Colombiana de Ciencias 107:201-222

Raut, S. K., and G. M. Barker. 2002. Achatina fulica Bowdich and other Achatinidae as pest in tropical agricultura. Pp. 55-114 in G. M. Barker (ed.). Mollusk as crop pest. CABI publishing. Hamilton, New Zealand. https://doi.org/ 10.1079/9780851993201.0055.

R Core Team. 2018. R: A language and environment for statistical computing. R foundation for statistical computing, Vienna, Austria. URL: www.R-project.org.

Shackleton, R. T., D. M. Richardson, C. M. Shackleton, B. Bennett, S. L. Crowley, K. Dehnen-Schmutz, and E. Marchante. 2019. Explaining people's perceptions of invasive alien species: A conceptual framework. Journal of Environmental Management 229:10-26. https://doi.org/10.1016/j.jenvman.2018.04.045.

Vázquez, A. A., J. Sánchez, E. Martínez, and A. Alba. 2017. Facilited invasion of an overseas invader: human mediated settlement and expansion of the giant African snail, Lissachatina fulica, in Cuba. Biological Invasions 19:1-4. https: //doi.org/10.1007/s10530-016-1266-3.

York, E. M., C. J. Butler, and W. D. Lord. 2014. Global decline in suitable habitat for Angiostrongylus (=Parastrongylus) cantonensis: the role of climate change. PloS ONE 9(8). https://doi.org/10.1371/journal.pone.0103831. 